\title{
RELAÇÃO ENTRE CULTURA ORGANIZACIONAL E ESTRATÉGIAS
}

\author{
M. F. DANJOUR, P. W. S. OLIVEIRA e M. E. M. AÑEZ \\ E-mail: brasil@ifrn.edu.br ${ }^{1}$; norte@ifrn.edu.br ${ }^{2}$
}

Artigo submetido em junho/2013 e aceito em fevereiro/2014

\section{RESUMO}

Este estudo apresenta como objetivo geral analisar as relações entre cultura organizacional e estratégias das Instituições de Ensino Superior do Estado do Rio Grande do Norte - IES/RN. Foi realizado um estudo do tipo explicativo predominantemente quantitativo com as 31 instituições. Os dados foram tratados por meio de um modelo preditivo de correlação canônica. Os resultados indicaram a existência de um estilo cultural empreendedor e uma tipologia estratégica prospectiva como predominantes, os resultados da correlação canônica indicaram uma forte relação entre as variáveis analisadas, de forma que se pôde identificar que $77,88 \%$ da variação do composto linear (estratégia organizacional) da primeira função são influenciados pela variação do outro composto (cultura organizacional) que a compõe. Por fim, conclui-se que o presente estudo confirma as relações existentes entre os estilos de cultura organizacional e as tipologias das estratégias organizacionais das IES estudadas.

PALAVRAS-CHAVE: Cultura Organizacional, Estratégia Organizacional, Instituição de Ensino Superior.

\section{RELATIONSHIP BETWEEN ORGANIZATIONAL CULTURE AND STRATEGIES}

\begin{abstract}
This study presents as objective to establish the causal relationships between culture and organizational strategies adopted by the Higher Education Institutions (HEIs) of the Rio Grande do Norte state. It was a study of the explanatory type, predominantly quantitative with the thirty-one institutions. The data were treated by means of a predictive model of canonical correlation. The results indicated the existence of an entrepreneurial cultural style and a prospective strategic outlook as the
\end{abstract}

predominant type. The canonical correlation results indicated a strong relationship between the variables, so that it was possible to identify that the $77.88 \%$ linear variation of the compound (organizational strategy) of the first function was influenced by the variation of another compound (organizational culture) that make it up. Finally, it can be concluded that this study confirms the relationship between the styles of organizational culture types and organizational strategies of the HEls.

KEYWORDS: Organizational Culture, Organizational Strategy, Higher Education Institution. 


\section{INTRODUÇÃO}

A cultura organizacional passa a ser considerada e aplicada ao mundo dos negócios com o surgimento da abordagem comportamental das organizações que se propõem a estudar as manifestações sociais do indivíduo e sua forma de interferência em um contexto organizacional.

Desta forma, o conceito de cultura organizacional segundo Morgan (1996), refere-se primordialmente aos hábitos e comportamento de um ou mais grupos de indivíduos que compõem a instituição.

Sendo estes indivíduos, parte fundamental do processo vital de uma organização, pode-se afirmar que estes são influenciados e influenciarão todas as dimensões da vida organizacional, o que tem levado os gestores, em um contexto de crescente turbulência e mudanças organizacionais, a desenvolverem um padrão de cultura que permita aos indivíduos terem uma postura proativa favorecendo a participação ativa em todos nos processos de mudanças (SCHNEIDER, 1996).

A estratégia, que saiu das fileiras militares, passou a ocupar lugar de destaque no universo organizacional através da lógica de planejamento estratégico, instrumento este capaz de definir o posicionamento da organização e estabelecer ações efetivas considerando os seus objetivos, frente aos ambientes interno e externo (ALMEIDA, 1991).

Com a evolução organizacional, observa-se um desenho ambiental bem diferente do vivenciado em meados do século passado. As mudanças vêm se apresentando de forma rápida e desordenada (HAMEL, 2000). Devido ao processo de internacionalização da economia, as distâncias entre as nações vêm se reduzindo e as transações, via rede vem levando as organizações, tanto na esfera privada quanto na esfera pública a desenvolverem suas estratégias considerando a competição global, para só assim, conseguirem se posicionar em um mundo onde a velocidade das mudanças é que determina as diretrizes das organizações.

Assim, observa-se que a organização, diferentemente do que se era trabalhado em uma lógica de relativa estabilidade, na qual predominava a linearidade e a centralização, por uma questão de sobrevivência é obrigada figurar com sua estrutura alinhada as suas estratégias. Desta forma os gestores organizacionais necessitam visualizar de forma sistêmica as interações dos diversos setores de sua organização com o seu ambiente externo, passando assim a desempenhar o seu papel como agentes ativos no processo de mudança organizacional (MINTZBERG, 2003).

Por conseguinte, enfatiza-se que em um contexto ambiental no qual o termo velocidade é uma expressão que significa sobrevivência com crescimento ou em outro extremo significa extinção das organizações, a sociedade vem se articulando e se conscientizando tanto em relação ao seu papel como usuário-cliente, quanto também em relação ao papel das organizações públicas e privadas como prestadores de serviços essenciais a sociedade (TONNI, 2009).

Assim, para atender essas novas demandas da sociedade no papel de cliente, tanto a iniciativa privada quanto a administração pública vem agregando as suas práticas administrativas, novos modelos e programas de gestão, que resultem na ampliação de mercado e ganho de competitividade para as empresas privadas e para organizações públicas esses avanços gerenciais 
necessitam resultar em redução dos custos operacionais e aumento da eficácia dos seus resultados.

Nessa busca por ganho de competitividade e aumento de eficácia o presente estudo destaca o atual momento de expansão vivenciado pelas organizações de ensino superior no país. Desta forma, observa-se segundo dados do INEP (2009), um significativo aumento no número de Instituições Ensino Superior - IES no país entre os anos de 1997 e 2007 totalizando 2.281 IES.

Esse quantitativo de IES foi dividido pela pesquisa INEP (2009, p. 8) de acordo com as características administrativa de cada instituição o que resultou em valores percentuais de " $90 \%$ de instituições privadas e $10 \%$ de instituições públicas, divididas entre federais (4,1\%), estaduais $(3,6 \%)$ e municipais $(2,7 \%)$ ", considerando todas as instituições que oferecem graduação nas modalidades presencial e distância.

Ao contextualizar os números do ensino superior no Estado do Rio Grande do Norte - RN, segundo dados do INEP (2009) no ano de 2000 esse estado tinha registrado junto ao Ministério da Educação - MEC um total de 08 IES cadastradas. Esses números de IES no estado do RN de acordo com dados do E- MEC (2010) aumentaram para 45 IES que desenvolvem suas atividades tanto na modalidade presencial quanto a distância. Esse aumento no número de IES no estado do RN se deu acompanhando a tendência nacional de expansão do ensino superior no Brasil.

Assim, é importante afirmar a necessidade das IES que atuam no estado do RN em buscar diferenciais na condução dos esforços gerenciais para prestar serviços educacionais com baixos custos de operações, mas que gerem eficácia nos seus resultados. Assim Mintzberg e Rose (2003, p. 272, 287) afirmam que as Instituições de Ensino Superior "são organizações que respondem continuamente às demandas ambientais porque existem muitos atores internos capazes de respostas independentes, sendo estas capazes de reagir aos interesses, demandas e pressões da comunidade que a rodeia".

Para tal fim, é necessário que os gestores dessas IES passem a conhecer os seus padrões culturais para que só assim sejam capazes de desenvolverem estratégias com o poder de integrar as necessidades internas das organizações com as exigências externas de seu ambiente (VIZEU E GONÇALVES 2010).

Neste contexto de transformações vivenciadas nas instituições de ensino superior em todo país o presente estudo busca responder a seguinte questão central de pesquisa: Qual a relação existente entre a cultura organizacional e estratégias das Instituições de Ensino Superior do Estado do Rio Grande do Norte?

Como objetivos específicos este estudo busca: identificar a tipologia de cultura organizacional presente nas IES/RN a partir do modelo dos estilos de funcionamento organizacional de Paz e Mendes; identificar o comportamento estratégico adotado pelas organizações estudadas tomando por base o modelo de Miles e Snow; estabelecer as relações causais entre a cultura e as estratégias organizacionais adotadas nas IES/RN.

\section{RELACIONANDO CULTURA E ESTRATÉGIA ORGANIZACIONAL}


Entendem-se, que os diferentes níveis da cultura da organização expressam um conjunto de valores que foram construídos ao longo da história da empresa, que foram desenvolvidos no seu processo de adaptação interna e externa. A cultura tem raízes profundas que, de certa forma, representam o DNA da organização (DUTRA; FLEURY; RUAS, 2006).

Dentro dessa perspectiva Porter (1986), ressalta a importância de adicionar o conhecimento a respeito das crenças e de outras características culturais às ferramentas gerenciais aplicadas as estratégias de negócios. Ele afirma ainda ser fortuito acrescentar que o saber acerca da cultura da organização é desejável para o estabelecimento de condições organizacionais apropriadas para bem administrar as mudanças decorrentes de estratégias, seus impactos e ressonâncias.

Desta forma, Schein (2009) reconhece a importância da cultura corporativa na gestão organizacional. Este autor destacar a influência dos elementos culturais na maneira como a empresa opera, as formas como as pessoas se comportam e no estabelecimento das estratégias da organização.

Carvalho e Ronchi (2005 p.17) mencionam que "[...] a cultura move a organização para uma direção, correta ou não". E acrescenta: "[...] provavelmente uma estratégia que esteja em desacordo com a cultura da organização dificilmente será implementada, e quando a for, fatalmente não alcançará sucesso". A cultura pode causar resistência ou favorecer as mudanças estratégicas na organização.

Segundo Mintzberg; Ahistrand e Lampel (2000), as literaturas apresentam uma variedade de ligações entre os conceitos de cultura e estratégia. Os autores resumiram algumas dessas ligações considerando a forma como foram desenvolvidas na literatura:

A. Estilo de tomada de decisão. Cada organização tem sua maneira peculiar de ser e de fazer as coisas. Na hora de decidir essa peculiaridade influencia a forma de pensar, de analisar sobre determinado assunto indicando assim que as concepções e direcionamentos estratégicos são significativamente influenciados pela cultura organizacional. Nas palavras dos autores a cultura "estabelece as premissas das decisões das pessoas". (MINTZBERG; AHISTRAND E LAMPEL, 2000, p. 197).

B. Resistência a mudanças estratégicas. A forma antiga, enraizada, de fazer e de decidir, o compromisso com as crenças existentes, cria uma zona de conforto difícil de ser abandonada pelos membros de uma organização, dificultando mudanças na estratégia. "Antes que o aprendizado estratégico... possa ocorrer, a lógica antiga [dominante] precisa ser, de certa forma, desaprendida pela organização...". (MINTZBERG; AHISTRAND E LAMPEL, 2000, p. 198).

C. Superar a resistência às mudanças estratégicas. Este ponto desperta para o risco de se ficar estagnado devido a uma cultura resistente. Mostra a necessidade de quem dirige a empresa aceitar e incorporar na cultura da organização a importância do novo, da flexibilidade. Mintzberg; Ahistrand e Lampel (2000) sugerem diversas formadas de se lutar contra esta realidade, inclusive com a nomeação de um alto executivo sem cargo, para fazer questionamentos e dar ideias, diretores externos para elaborarem perguntas a respeito das crenças em tempos de mudanças, etc.

Mitzberg, Ahistrand e Lampel (2000 p. 199) dizem que: "mudanças radicais na estratégia precisam ser baseadas em mudanças fundamentais na cultura". Essa compreensão mantém a lógica da inter-relação entre a organização e a sua cultura, ou até mesmo, entendendo a 
organização como a própria cultura. De uma forma ou de outra pode-se perceber quatro fases em que isto acontece no processo de mudança organizacional:

Quadro 01: Mudança Estratégicas X Mudanças Culturais

\begin{tabular}{|c|c|}
\hline Deriva estratégica & $\begin{array}{c}\text { Mudanças radicais são precedidas por um alargamento do } \\
\text { vazio entre as crenças organizacionais e as características } \\
\text { do ambiente. }\end{array}$ \\
\hline Descongelamento dos sistemas de crenças correntes & $\begin{array}{c}\text { Nesta situação, crenças organizacionais anteriormente } \\
\text { inquestionadas são expostas e contestadas. }\end{array}$ \\
\hline Experimentação e reformulação & $\begin{array}{c}\text { Período pós confusão. Desenvolvimento de uma nova } \\
\text { visão estratégica. }\end{array}$ \\
\hline Estabilização & $\begin{array}{c}\text { Fase da acomodação, do comprometimento dos membros } \\
\text { da organização com novos sistemas de crenças que } \\
\text { pareçam funciona. }\end{array}$ \\
\hline
\end{tabular}

Fonte: Adaptado de Mintzberg; Ahistrand e Lampel (2000)

D. Valores Dominantes. Nesta consideração é colocada em foco a vantagem competitiva de uma empresa baseado nos seus pontos fortes exemplificados como: "atendimento, qualidade e inovação". Essas vantagens competitivas são usadas pelas empresas "para sustentar perspectivas estratégicas notavelmente estáveis".

E. Choque de Culturas. O autor apresenta este fator como um motivo de desunião de empresas que tentam fundir-se. $O$ fato de não existir culturas organizacionais exatamente iguais e de cada empresa ter a sua forma de ler o ambiente e de reagir frente aos mesmos fatos cria uma zona de tensão, na maioria das vezes, impossível de ser solucionada.

Diante da força, exercida pela cultura, no mundo organizacional, pode ser relevante para os líderes entender, cada vez mais, sobre a sua cultura, para onde ela está conduzindo os movimentos da instituição e se a mesma favorece ou bloqueia o estabelecimento de novos modelos estratégicos.

Assim, Schein (2009) afirma que uma estratégia genérica tem por fim orientar os esforços dos indivíduos nas organizações em busca de um objetivo compartilhado, que fica possivelmente na dependência da cultura organizacional. A cultura por sua vez, é a identificação da organização na medida em que os valores compartilhados pelos indivíduos tornam-se pressupostos inconscientes que determinaram a forma como o grupo percebe, sente, pensa e age.

Por sua vez, Vizeu e Gonçalves (2010) estabelecem três pontos relevantes à discussão sobre a relação cultura e estratégia.

O primeiro deles é reconhecer o papel da liderança fortemente simbólico uma vez que essa desenvolve e molda crenças, valores e suposições, direcionam as experiências de aprendizado dos membros e provocam mudanças nas crenças, valores e suposições ao longo do tempo na organização (SCHEIN, 2009). Schein (2009, p. 213), afirma que "os fundadores têm maior impacto como o grupo inicialmente define e soluciona seus problemas de integração interna e de adaptação externa", desta forma observa-se nesta discussão um forte direcionamento dos fundadores e primeiros líderes de uma organização na forma de agir e pensar estrategicamente a organização.

O segundo consiste na definição de capital social como sendo rede de relacionamento, que uma pessoa possui, desta forma o autor afirma que quanto maior for à rede de relacionamento que a organização na figura dos seus líderes tiver com os diversos segmentos 
organizacionais, maior será a compreensão do ambiente e das suas variáveis que influenciam significativamente as decisões estratégicas da organização.

O último ponto destacado pelos autores é que à cultura e a estratégia estão relacionadas às diferentes visões existentes na organização.

Com base nas visões propostas anteriormente acerca da discussão relacionada à cultura e estratégia organizacional é possível a criação de uma relação baseada nos estilos culturais propostos por Paz e Mendes (2009) e as tipologias de estratégias genéricas de Miles e Snow (2003).

Assim, a figura 02 apresenta em cada tipo de configuração organizacional um estilo cultural e uma tipologia de estratégia adequada para aquela cultura.

Quadro 2: Configurações organizacionais: aliando culturas e estratégias

\begin{tabular}{|l|l|l|l|l|}
\hline \multicolumn{1}{|c|}{$\begin{array}{c}\text { Relações Cultura x } \\
\text { Estratégias }\end{array}$} & \multicolumn{1}{|c|}{ Relação 01 } & Relação 02 & \multicolumn{1}{|c|}{ Relação 02 } & \multicolumn{1}{c|}{ Relação $\mathbf{0 2}$} \\
\hline $\begin{array}{l}\text { Estilos Culturais } \\
\text { Paz e Mendes (2008) }\end{array}$ & Empreendedor & Burocrático & Afiliativo & Individualista \\
\hline $\begin{array}{l}\text { Estratégias Genéricas } \\
\text { Miles e Snow (2003) }\end{array}$ & Prospectiva & Defensiva & $\begin{array}{l}\text { Analisadora } \\
\text { (Prospectiva ou } \\
\text { Defensiva) }\end{array}$ & Reativa \\
\hline
\end{tabular}

Fonte: Adaptado de Danjour (2010, p.45).

Ao observar a figura 2 identificam-se os seus estilos culturais e respectivas tipologias de estratégias genéricas. Para construção da referida figura observou-se a percepção de Mintzberg; Ahistrand e Lampel (2000, p. 234) que afirmam que "as tipologias de Miles e Snow se reduz a duas formas básicas, com a terceira sendo uma forma híbrida e a quarta uma coleção de respostas inadequadas." Tomando por base o alinhamento prévio feito pelo Mintzberg buscou-se relacionar os estilos culturais propostos por Paz e Mendes (2009) com as tipologias estratégicas de Miles e Snow (2003).

\section{ESTUDOS ACERCA DE CULTURA E ESTRATÉGIA}

Neste momento serão discutidos os resultados de algumas dissertações e artigos científicos objetivando identificar no estado da arte, novas perspectivas para as discussões desencadeadas ao longo do presente trabalho.

Degenhardt (2006) estudou as estratégias competitivas, as culturas organizacionais e o relacionamento entre elas, na indústria mecânica brasileira de equipamentos pesados, o seu estudo utilizou como modelos teóricos os instrumentos produzidos por Cameron e Quinn (cultura organizacional) e Miles e Snow(diagnóstico de estratégia).

Desta forma, Degenhardt (2006) apresenta como resultados conclusivos de sua pesquisa que $71 \%$ das empresas utilizam a estratégia defensiva, segunda estratégia mais utilizada com $21 \%$ foi a analisadora. No que diz respeito ao diagnóstico de cultura organizacional observou-se em $50 \%$ das empresas estudadas uma tipologia cultural denominada de racional que se orienta ao meio ambiente a ao controle interno cujo seu foco é alcançar um desempenho superior cumprimento de metas e as demais empresas apresentam uma diversidade entre a cultura hierárquica e grupal. Por fim Degenhardt (2006) conclui o seu estudo afirmando que foi possível 
estudar a cultura organizacional e os seus relacionamentos com a estratégia competitiva no setor pesquisado, porém sem sincronia preconizada pelo modelo adotado.

Em sua pesquisa Moreira (2007) que apresenta como objetivo geral estudar as relações existentes entre os perfis estratégicos com performance organizacional alcançada pelas Instituições de Ensino Superior -IES privadas na região sul do pais. Para realizar esta pesquisa a autora utilizou o modelo de análise de estratégias genéricas proposto por Miles e Snow (1978) para identificar o perfil estratégico das organizações estudadas, para a mensuração da performance, foi utilizado o modelo de Kelm (2003).

Os resultados da pesquisa de Moreira (2003), ao analisar as tipologias estratégicas das IES, identificaram a predominância das estratégias defensiva $(36,7 \%)$, seguida da prospectiva (25\%), analisadora (23,3\%) e reativa (15\%). No tocante à análise de performances das IES, identificou-se a presença dos 29 indicadores presentes no modelo do BSC de Kelm (2003). Quando comparados as tipologias estratégicas aos indicadores identificou-se a existência significativa de relação entre as tipologias estratégicas com os indicadores de performance de desempenho dos estudantes, taxa de evasão, projeto de extensão, projetos com financiamento externo, volume de projetos com parceiros externos, qualidade de aula, volume de publicação de professor com aluno, relação de professor aluno e qualidade das aulas.

Relacionando cultura organizacional com o processo de formulação de estratégias Green (1988) em seu estudo apresenta uma alternativa para reconstruir a visão estática formada pelos gestores organizacionais acerca das relações entre cultura e estratégia. Desta forma o autor apresenta como problema dessa relação estática uma cultura engessada em hábitos, que provocam resistências ao processo de mudança organizacional e acaba por frustrar a formulação e implementação das estratégias. Desta forma, o seu estudo redefiniu a cultura como tendo um significado compartilhado de forma coletiva proporcionando um novo sentido as ações dos líderes, sedo a estratégia um conjunto de significados e a gestão estratégica um processo cultural, que permite os gestores perceberem de forma diferente a sua organização, compreendendo tanto a natureza e os propósitos fundamentais da organização, quanto o seu papel dentro dela.

Por fim, Shrivastava (1985) apresenta a cultura organizacional como um conjunto de sistemas compartilhados que dão significado aos acontecimentos e situações vivenciadas nas organizações. Desta forma, o autor apresenta em seu estudo quatro elementos que manifestam o resultado da interatividade entre esses sistemas que são os mitos, sistemas de linguagem, símbolos e sistemas de valores e normas de comportamento. Assim, esses elementos que constituem a cultura organizacional, desempenham uma forte influência sobre o processo de decisões estratégicas das organizações, substituindo a racionalidade dessas decisões.

Neste contexto, Shrivastava (1985) relaciona de forma sistêmica os elementos que constituem a cultura da organização ao processo decisório estratégica das organizações, onde apresenta os possíveis problemas provocados pela falta de alinhamento entre essas dimensões. Por fim, Shrivastava (1985) afirma as dificuldades de se modificar alguns dos elementos formadores da cultura, todavia o autor apresenta o gestor como responsável por desenvolver estratégias alinhadas à cultura da organização. 


\section{METODOLOGIA}

Esta pesquisa quanto aos seus objetivos é considerada como explicativa. Segundo Collis e Hussey (2005, p. 71) esse tipo de pesquisa "tem como objetivo entender os fenômenos, descobrindo e mensurando relações causais entre eles". Quanto aos procedimentos este estudo classifica-se com sendo uma survey analítica, que segundo Collis e Hussey $(2005$, p. 71$)$ é uma metodologia positivista que a partir de uma amostra representativa da população permite realizar inferências sobre esta, além de "determinar se há alguma relação entre diferentes variáveis", necessitando que o pesquisador identifique as variáveis dependentes e independentes do seu estudo. Por fim, quanto à natureza o estudo é quantitativo no que diz respeito à classificação das estratégias genéricas, tipologia de cultura organizacional e estabelecimento das possíveis relações entre estas variáveis.

Para esta pesquisa a população compreende as Instituições de Ensino Superior que atuam no estado do Rio Grande do Norte na figura do seu principal gestor em nível estratégico. Desta forma a população deste estudo foi composta por 31 gestores das 31 Instituições de Ensino Superior que possuem sede física no estado do Rio Grande do Norte. O estudo não utilizou amostra sendo considerado do tipo censitário.

Para efeito desta pesquisa foram utilizados um questionário com um total de 79 perguntas fechadas divididas em 5 questões que permite se identificar características gerais das instituições estudadas, 30 questões elaboradas com base nos modelos teóricos propostos por Paz e Mendes (2009) para identificar as tipologias culturais e 44 questões desenvolvidas com base no modelo teórico de Miles e Snow (2003) para avaliar estratégias genéricas existentes nas organizações estudadas.

Os dados coletados foram analisados com a utilização dos modelos de Paz e Mendes (2008) e Miles e Snow (2003). O instrumento desenvolvido por Paz e Mendes (2008) foi analisado observando as 30 variáveis propostas no questionário que utilizam uma escala de 5 pontos para avaliação da cultura organizacional do IFRN. Por sua vez, o instrumento para identificação das tipologias estratégicas conforme modelo de Miles e Snow(2003) foi adaptado do questionário desenvolvido por Conant, Monkwa e Varadajan (1990), no qual as assertivas presentes no questionário original foram transformadas em 44 afirmações que foram mensuradas por meio de escala Likert com 5 pontos com variação de 0 à 4 a exemplo do instrumento anterior. Assim, a apuração dos resultados para os dois instrumentos utilizados foram realizadas com o cálculo da média em relação a escala de 5 pontos com variação de 0 a 4 , no qual o ponto médio foi considerado como sendo 2 , sedo as medidas próximas a esse ponto consideradas como uma presença moderada de determinado estilo, enquanto acima de 2,5 o padrão foi considerado predominante e abaixo de 1,5 o padrão foi considerado pouco característico. Além da análise da média foi realizado ainda os testes de diferença entre as médias e analise dos desvios padrões.

As últimas análises realizadas neste trabalho buscou identificar as relações existentes entre cultura organizacional e as tipologias de estratégias presentes no IFRN. Para tal, foi utilizada a análise multivariada correlação canônica. Para Hair Jr. et al $(2009$, p. 3) a correlação canônica é um "modelo estatístico multivariado que facilita o estudo de inter-relações entre conjuntos de múltiplas variáveis dependentes e múltiplas variáveis independentes", em outras palavra a correlação canônica possibilita de forma simultânea determinar a correlação de múltiplas variáveis dependentes a partir de várias variáveis independentes. Para efeito desse 
estudo foram consideradas como variáveis dependentes as tipologias de estratégicas genéricas sendo elas: prospectiva, defensiva, analisadora e reativa. As variáveis independentes foram consideradas como sendo os estilos de cultura organizacional, sendo eles: empreendedor, afiliativo, individualista e burocrático. Todavia, para feito da análise de correlação canônica as variáveis ditas inicialmente dependentes e independentes assumem uma relação de reciprocidade.

Por fim, os resultados encontrados com a realização da correlação canônica foram confirmados com a utilização da técnica estatística de correlação de Pearson que segundo Collis e Hussey (2005) possibilita identificar e confirmar a medida de força de associação entre cada par de fatores de cultura e estratégia organizacional. A última análise realizada nesta pesquisa buscou relacionar as culturas e estratégias encontradas nas IES com uma proposta teórica apresentada na figura 2 da fundamentação teórica, identificando conformidades e inconformidades dos resultados em relação ao modelo proposto.

\section{ANÁLISE E DISCUSSÕES DOS RESULTADOS}

Os resultados gerais em média das IES estudadas para cultura organizacional indicam a existência de valores para os quatro estilos de funcionamento das organizações. Tais médias foram encontradas considerando todos os pontos recebidos por cada fator que formaram os quatro estilos culturais das organizações.

Tabela 1: Resultado geral em média para cultura organizacional

\begin{tabular}{l|r|r}
\hline ITENS & MÉDIA & DESVIO PADRÃO \\
\hline Estilo Empreendedor & 3,2823 & 0,5264 \\
\hline Estilo Afiliativo & 2,9770 & 0,6228 \\
\hline Estilo Individualista & 0,9539 & 0,5208 \\
\hline Estilo Burocrático & 1,9637 & 0,5033 \\
\hline
\end{tabular}

Fonte: Dados da pesquisa 2012

Desta forma, ao se analisar a tabela 1 observar-se os estilos empreendedor $(3,2823)$ e afiliativo $(2,9770)$ com as maiores médias. Considerando as médias superiores a 2,5 como sendo uma presença predominante da tipologia de cultura organizacionais. Assim, Paz, Mendes e Gabriel (2001) indicam à existência de IES que desenvolvem suas atividades como sistemas aberto, com um significativo grau de maturidade. Mesmo com a identificação de uma média elevada para o estilo afiliativo, pode-se observar que parte do grupo ainda está em fase de consolidação do comportamento, podendo com o acúmulo de experiências apresentarem o que Schein (2009) chama de mudança cultural, ou seja, resgatar no futuro, comportamentos baseados em estilos culturais mais amadurecidos.

Assim, essas organizações com o estilo empreendedor, caracterizam-se por valorizarem a inovação e a busca por relações de trocas equilibradas, valorizando e incentivando as iniciativas empreendedoras individuais e grupais, Todavia identifica-se ainda em alguns casos comportamentos organizacionais que geram o conformismo dos indivíduos manifestado por meio da plena aceitação das normas e regras, em troca de seguranças, vantagens e assistência, gerando em alguns momentos um forte envolvimento emocional do indivíduo com a organização (PAZ E MENDES, 2009). 
Ao analisar os resultados gerais em média das IES estudadas para as tipologias de estratégias organizacionais, observa-se a existência de valores para as quatro tipologias de estratégias organizacionais propostas por Miles e Snow (2003). Tais médias foram encontradas considerando todos os pontos recebidos por cada fator que formaram as quatro tipologias estratégicas das IES estudadas.

Tabela 2: Resultado geral em média para estratégia organizacional

\begin{tabular}{l|r|r}
\hline \multicolumn{1}{c|}{ ITENS } & \multicolumn{1}{c|}{ MÉDIA } & DESVIO PADRÃO \\
\hline Estratégia Prospectiva & 2,5249 & 0,5443 \\
\hline Estratégia Defensiva & 2,1642 & 0,4142 \\
\hline Estratégia Analisadora & 2,2698 & 0,4388 \\
\hline Estratégia Reativa & 2,0411 & 0,3982 \\
\hline
\end{tabular}

Fonte: Dados da pesquisa 2012

Desta forma ao se observar com a maior média a tipologia prospectiva (2,5249), considerando-se o ponto médio como sendo 2 e as médias superiores a 2,5 como sendo uma presença predominante da tipologia, pode-se identificar segundo Miles e Snow (2003) a predominância de organizações orientadas para a aprendizagem, com uma estrutura organizacional flexível, fluida e descentralizada o que Mintzberg (2003) denomina de adhocracia.

Além da estratégia predominante foi identificado ainda as três estratégias propostas por Miles e Snow com médias ente 2,0 e 2,5 o que se pode verificar uma presença moderada das outras estratégias. Desta forma identificou-se as estratégias analisadora $(2,2698)$, seguida da estratégia defensiva $(2,1642)$ e com a menor média a estratégia reativa $(2,0411)$. Essas médias permitem uma análise no sentido identificar a influência geral dessas tipologias estratégicas sendo como tipologia estratégica principal ou secundária no comportamento estratégico das IES do Estado do Rio Grande do Norte.

Assim, apesar de se identificar a predominância de uma determinada estratégia não se pode desprezar a presença das demais estratégias de forma que se tenha uma estratégia analisadora como estratégia predominante em uma organização e uma estratégia prospectiva ou defensiva como uma estratégia secundária com média no nível de presença moderada da tipologia. Desta forma, se apresenta uma estratégia analisadora com uma possibilidade de força prospectiva ou defensiva.

As últimas analises realizadas na pesquisa buscou identificar por meio das técnicas de correlação canônica e de correlação de Pearson as relações existentes entre os estilos de cultura organizacional propostos por Paz e Mendes (2009) e as tipologias estratégicas propostas por Miles e Snow (2003).

Assim, para a correlação canônica, os fatores preditivos ou explicativos foram compostos pelos estilos culturais empreendedor, afiliativo, individualista e burocrático, por sua vez, o conjunto explicado será composto pelas tipologias estratégicas prospectivas, defensiva, analisadora e reativa.

Tabela 3: Correlação Canônica e Teste de Significância

\begin{tabular}{r|r|c|r|r|r|r} 
Função Canônica & Correlação Canônica R & \multicolumn{1}{c|}{$\mathbf{R}^{\mathbf{2}}$} & Qui-quadrado & GL & \multicolumn{1}{c|}{ Sig. } & \multicolumn{1}{c}{$\boldsymbol{\lambda}$ de Wilks } \\
\hline 1 & 0,8825 & 0,7788 & 42,3128 & 16 & 0,0004 & 0,1903 \\
\hline 2 & 0,3489 & 0,1218 & 3,8391 & 9 & 0,9217 & 0,8602 \\
\hline
\end{tabular}




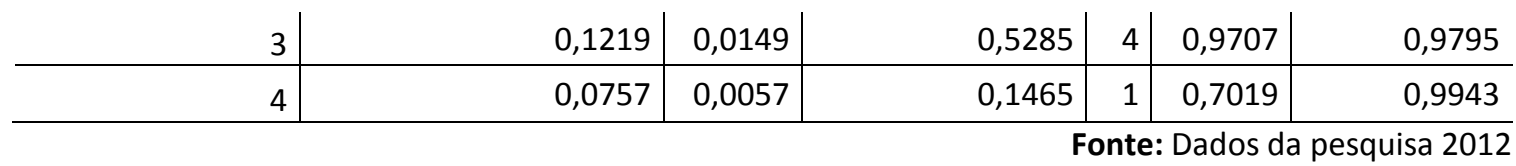

Para a escolha das funções canônicas que serão interpretadas, Hair Jr (2009, p. 5) propõem que se analise três critérios fundamentais que são: “(1) o nível de significância estatística da função, (2) magnitude da correlação canônica e (3) medida de redundância para o percentual de variância explicada a partir dos dois conjuntos de dados"

Desta forma, observa-se que a correlação canônica permite o pesquisador mensurar a força de relação associativa entre dois conjuntos de variáveis. Assim, a tabela 3 apresenta quatro funções canônicas dentre estas funções foi escolhida a primeira devido a esta ser significante a $1 \%$ na qual se identificou um p-valor de 0,0004 , o outro fator observado foi R canônico de 0,8825 que indica uma forte correlação positiva e significativa. Quando elevado ao quadrado, tal coeficiente representa a quantidade de variância em um composto linear da função canônica atribuída a outro composto da mesma função sendo este o $R^{2}$ canônico. Assim, pode-se afirmar que, $77,88 \%$ da variação em um composto linear da primeira função são influenciados pela variação no outro composto que a compõe.

Observando o percentual de $77,88 \%$ de influência que o segundo composto linear (culturas) exerce sobre o primeiro composto (estratégias) presentes na primeira função, pode-se afirmar que existe um significativo grau de influência da cultura organizacional no posicionamento estratégico das IES estudadas, dessa maneira as influencias serão identificadas quando analisadas as cargas canônicas e a correlação de Pearson.

Tabela 4: Índices de redundância

\begin{tabular}{l|r|c|r}
\hline \multicolumn{1}{c|}{ Variáveis } & Variância Compartilhada & $\mathbf{R}^{\mathbf{2}}$ & Redundância \\
\hline Dependentes & 0,3026 & 0,7788 & 0,2356 \\
\hline Independentes & 0,6272 & 0,7788 & 0,4885 \\
\hline \multicolumn{4}{c}{ Fonte: Dados da pesquisa 2012 }
\end{tabular}

Em relação a Tabela 4 que apresenta os índices de redundância de acordo com a proposta de análise de Hair Jr (2009), observou-se que os valores são representativos onde $23,56 \%$ de variância nas variáveis dependentes é explicada pela variável estatística canônica independente e $48,85 \%$ de variância nas variáveis independentes é explicada pela variável estatística canônica dependente.

Tabela 5: Cargas canônicas

\begin{tabular}{l|r} 
Variáveis Dependentes & Cargas Canônicas \\
\hline$Y_{1}-$ Prospectiva & 0,9573 \\
\hline$Y_{2}$ - Defensiva & 0,0465 \\
\hline$Y_{3}$ - Analisadora & 0,4188 \\
\hline$Y_{4}$ - Reativa & 0,3410 \\
\hline Variáveis Independentes & \\
\hline$X_{1}$ - Empreendedor & 0,8922 \\
\hline$X_{2}-$ Afiliativo & 0,6871 \\
\hline$X_{3}-$ Individualista & $-0,7866$ \\
\hline$X_{4}-$ Burocrático & $-0,7887$ \\
\hline
\end{tabular}


Fonte: Dados da pesquisa 2012

A partir da Tabela 5 podem-se identificar as cargas canônicas ou correlações estruturais canônicas. Segundo Hair Jr (2009, p. 7) esse índice "mede a correlação linear simples entre uma variável observada original no conjunto dependente ou independente e a variável estatística canônica do conjunto". Desta forma por se tratar de uma técnica preditiva a análise será conduzida observando uma escala variando de -1 a 1 , no qual as medidas mais próximas do 0 indica uma influência fraca de determinado fator, e mais próximo de -1 ou 1 indica uma influência forte de determinado fator em relação ao outro. Tomando por base os critérios de análise utilizados por Puente-Palacios (1995) o presente estudo considerou uma carga canônica igual ou superior a 0,4 para as interpretações de todas as correlações.

Desta forma, observa-se como primeiro grupo as quatro variáveis dependentes que são as tipologias estratégicas propostas por Miles e Snow (2003) que podem ser identificadas como Y1 prospectiva, Y2 defensiva, Y3 analisadora, Y4 reativa. O segundo grupo é composto pelas quatro variáveis independentes que são os estilos culturais propostos por Paz e Mendes (2009) que se identificam como X1 empreendedor, X2 afiliativo, X3 individualista e X4 burocrático.

Ao se analisar a tabela 5 observa-se que o estilo de cultura empreendedor $(0,8922)$ influência fortemente e positivamente a estratégia prospectiva $(0,9573)$ exercendo ainda uma influência moderada e positiva sobre a estratégia analisadora $(0,4188)$.

Essas influências colaboram com a proposta teórica na qual apresentam esse fator cultural teoricamente relacionado a essas tipologias estratégicas, desta maneira pode-se retomar as relações teóricas discutidas ao longo da fundamentação teórica na qual Paz e Mendes (2009) apresentam o estilo cultural empreendedor com características favoráveis ao desenvolvimento de competências, incentivo a inovação e ao crescimento profissional, sendo este padrão cultural favorável ao desenvolvimento de estratégia como do tipo prospectiva, sendo identificada como uma estratégia favorável ao aprendizado, fluida e descentralizada, ou seja, o ambiente advindo de um estilo cultural empreendedor vai favorecer o desenvolvimento da estratégia prospectiva sendo está ralação a mais forte encontrada neste estudo.

Por sua vez, a tipologia estratégica analisadora é considerada por Mintzberg; Ahistrand e Lampel (2000) como uma tipologia intermediária estando entre as estratégias prospectivas e defensivas. Desta forma, a cultura empreendedora vai exercer esta influência moderada devido as características comuns que a estratégia analisadora possui em relação a estratégia prospectiva. Dentre essas características Miles e Snow (2003) destacam um rígido controle de custos conduzido com flexibilidade e adaptabilidade, além do equilíbrio de eficiência com aprendizagem. Desta forma, identificou-se uma influência moderada do estilo cultural empreendedor na tipologia estratégica analisadora.

O estilo cultural afiliativo $(0,6871)$ apresenta uma forte influência positiva sobre a tipologia estratégica prospectiva $(0,9573)$, exercendo ainda, uma influência moderada e positiva sobre a estratégia analisadora $(0,4188)$.

Estando o estilo cultural afiliativo em uma fase de maturidade que antecede o estilo empreendedor, Paz e Mendes (2003) apresentam esse estilo com características coletivistas. A organização com este estilo cultural mantém uma relação de troca com seus funcionários, onde oferece vantagens e segurança em troca da aceitação das normas e regras estabelecidas pelo grupo sem questionamentos. Desta forma, apesar da existência de um processo rígido de regras 
e normas impostas pelos membros destas organizações, esse estilo cultural vai exercer forte influência positiva na estratégia prospectiva, apresentando-se dentro do esperado, uma vez que, esse estilo cultural favorece a existência de um ambiente coletivista propício ao desenvolvimento tanto dessas organizações quanto dos seus membros (PAZ e MENDES 2003).

Todavia, conceitualmente a tipologia estratégica mais favorável a ser desenvolvido nesse estilo cultural é a estratégia analisadora, sendo esta identificada na tabela 4 como recebendo uma influência moderada do estilo cultural afiliativo. Essa influência moderada pode-se justificar pelo perfil inovador das Instituições de Ensino Superior do Estado do RN que se apresentam em um ambiente de relativas mudanças o que favorece e existência de uma maior influência do estilo cultural afiliativo sob a estratégia prospectiva (DAFT 2002).

Já o estilo cultural individualista $(-0,7866)$ exerce uma forte influência negativa sobre a estratégia prospectiva $(0,9573)$ e uma influência moderada e negativa sobre a tipologia estratégia analisadora $(0,4188)$.

Essa influência forte e negativa, identifica-se devido as diferenças conceituais entre essa duas varáveis, desta forma, Paz e Mendes (2009) apresentam o estilo cultural individualista como sendo propício para o desenvolvimento de um ambiente organizacional pouco maduro, no qual os interesses individuais dos seus membros estão acima das necessidades mais profissionais, 0 que vai de encontro ao desenvolvimento das estratégias prospectivas e analisadoras, que segundo Daft (2002) são estratégias focadas no desenvolvimento tanto das organizações quanto de seus membros, favorecendo o aprendizado e a criatividade.

O último estilo cultural, no qual foi analisando a carga canônica, foi o burocrático $(0,7887)$ que exercer uma influência forte e negativa sobre a estratégia prospectiva $(0,9573)$ e uma influência moderada e negativa sobre a tipologia estratégica analisadora $(0,4188)$.

Assim como o estilo cultural individualista, o estilo burocrático vai exercer uma forte influência negativa sobre a estratégia prospectiva devido as suas características que exaltam sistemas rígidos de controle, uma forte estrutura hierárquica com a centralização do poder decisório no topo da estrutura organizacional (PAZ E MENDES, 2009). Desta forma, esse padrão cultural não é favorável para o desenvolvimento de uma estratégia prospectiva que necessita de um estilo cultural favorável a aprendizagem que comportem uma estrutura organizacional flexível e descentralizada.

Todavia, foi identificada uma influência moderada e negativa da cultura burocrática na tipologia estratégica analisadora. Essa relação pode ser explicada devido a uma tendência de proximidade existente entre as estratégias prospectivas e analisadoras das IES distanciando essas características da estratégia defensiva, o que permite essa influência moderada e negativa exercida pela cultura burocrática.

Como forma de reafirmar os resultados identificados no modelo preditivo de correlação canônica, foram analisados na tabela 5 os índices de correlação de Pearson, existentes entre os estilos culturais e as tipologias estratégicas das IES do RN. Diferentemente da análise da correlação canônica, a correlação de Pearson permite identificar apenas a significância da correção com a visualização do p-valor e a identificação da força da correlação, não sendo possível afirmar a existência de múltiplas influencias entre os fatores analisados, como foi realizado nas discussões da correlação canônica. 
Tabela 5: Correlação de Pearson

\begin{tabular}{|c|c|c|c|c|c|}
\hline & & Prospectiva & Defensiva & Analisadora & Reativa \\
\hline \multirow{3}{*}{ Empreendedor } & Correlação de Pearson & $0,7639 * *$ & 0,0149 & 0,3235 & 0,2176 \\
\hline & Significância & 0,0000 & 0,9368 & 0,0759 & 0,2397 \\
\hline & $\mathrm{N}$ & 31 & 31 & 31 & 31 \\
\hline \multirow{3}{*}{ Afiliativo } & Correlação de Pearson & $0,5937 * *$ & $-0,0100$ & 0,2674 & 0,1628 \\
\hline & Significância & 0,0004 & 0,9574 & 0,1458 & 0,3816 \\
\hline & $\mathrm{N}$ & 31 & 31 & 31 & 31 \\
\hline \multirow{3}{*}{ Individualista } & Correlação de Pearson & $-0,6265 * *$ & 0,0904 & $-0,1294$ & $-0,1847$ \\
\hline & Significância & 0,0002 & 0,6286 & 0,4878 & 0,3199 \\
\hline & $\mathrm{N}$ & 31 & 31 & 31 & 31 \\
\hline \multirow{3}{*}{ Burocrático } & Correlação de Pearson & $-0,6706 * *$ & $-0,1122$ & $-0,3830 *$ & 0,3363* \\
\hline & Significância & 0,0000 & 0,5480 & 0,0335 & 0,0500 \\
\hline & $\mathrm{N}$ & 31 & 31 & 31 & 31 \\
\hline
\end{tabular}

Desta forma, considerando-se um nível de significância a 1\%, a tabela 5 apresenta uma forte correlação positiva entre os estilos culturais empreendedor e afiliativo, com a tipologia estratégia prospectiva e uma forte correlação negativa entre a estratégia prospectiva e os estilos culturais individualistas e burocráticos. Esses resultados reafirmam os identificados na análise das cargas canônica e reafirmam a existência de relações entre a cultura e a estratégia organizacional.

Outros índices de correlações de Pearson, significantes a 5\%, que reafirmam as relações encontradas na análise de correlação canônica, foram as correlações entre o estilo cultural burocrático e as estratégias analisadoras e reativas. Sendo uma correlação negativa e moderada entre o estilo cultural burocrático e a estratégia analisadora, e uma correlação positiva e moderada entre o estilo cultural burocrático e a estratégia reativa.

Todavia, é importante reafirmar que a análise de correção de Pearson permite a correlação linear entre cada fator estudado, diferentemente da correlação canônica, que assim como na técnica estatística de regressão múltipla, permite-se afirmar as múltiplas relações entre as variáveis dependentes e independentes analisadas, ou seja, o modelo preditivo de correlação canônica permitiu identificar a influência da cultura no processo de definição da tipologia de estratégia genérica adotadas pelas IES do RN.

Em relação a figura 3, foram listados os estilos culturais e suas respectivas estratégias em cada IES do estado, de acordo com as maiores médias identificadas para cada fator. Análise desta figura será realizada baseando-se no modelo teórico (figura 2) proposto na fundamentação teórica com o alinhamento das tipologias de estratégias de Miles e Snow (2003) com os estilos culturais propostos por Paz e Mendes (2008). A partir dessa relação procurou-se desenvolver uma comparação para atestar nas IES do RN as conformidades e desconformidades das relações entre os seus estilos culturais e suas respectivas estratégias. 
Esta análise serve de complemento aos resultados identificados na correlação canônica que identificou estatisticamente a influência da cultura nas estratégias desenvolvidas pelas organizações estudadas.
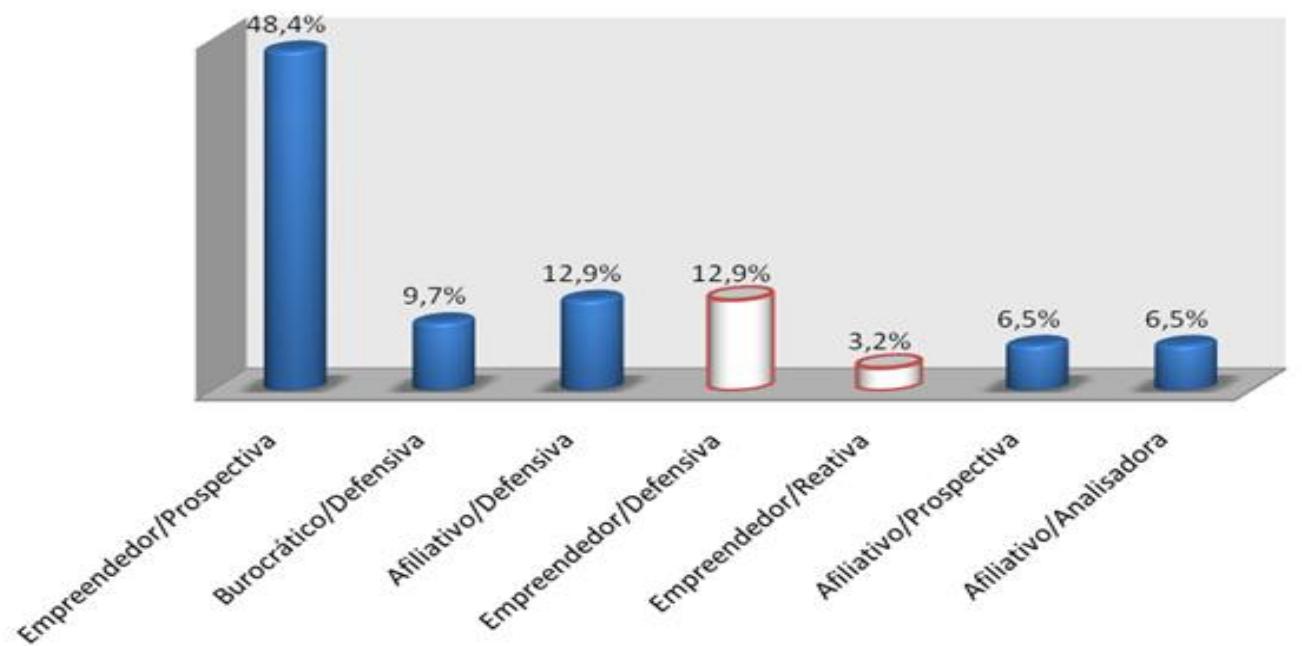

Gráfico 1: Incidências das tipologias de cultura e estratégias em conformidade com o modelo teórico proposto. Fonte: Dados da pesquisa 2010.

Ao se observar a figura 03 identificou-se que a soma dos percentuais encontrados para cada estilo cultural e estratégia organizacional, por IES, formam $84 \%$ de conformidades com o modelo teórico proposto na figura 02 do referencial teórico e apenas $16 \%$ estão em desconformidade com a proposta teórica.

Esse nível de conformidades está satisfatório ao se comparar com a pesquisa desenvolvida por Degenhardt (2006) no qual buscou identificar o relacionamento entre cultura e estratégia organizacional a partir dos modelos Quinn e Rohrbaugh (cultura) e de Miles e Snow (estratégia organizacional), a análise nesse caso foi desenvolvida com base no modelo de Quinn e Grath (1985). Assim, Degenhardt (2006) ao investigar as relações entre cultura e estratégia na indústria brasileira de mecânica pesada identificou a ocorrência de $57 \%$ de conformidades contra $43 \%$ de desconformidade com o modelo proposto.

Desta forma, identificou-se no presente estudo um significativo percentual de conformidade entre as bases teóricas propostas na figura 2 com os resultados encontrados no presente estudo o que reafirmam os resultados da correlação canônica.

Todavia, é importante reafirmar a postura predominantemente inovadora, flexível e focada no processo de aprendizagem, identificada nas IES no estado do RN, uma vez que se identificou na figura 3 um percentual de 48,4\% de predominância da cultura empreendedora associada a estratégia prospectiva nas IES, estado também o estilo cultural afiliativo associado as estratégias prospectiva e analisadora contribuindo com essa análise.

Em relação ao percentual de não conformidades identificadas na figura 3, pode ser justificada como uma possível falta de compreensão da administração em relação as possibilidades que $o$ estilo cultural presente em sua organização proporciona ao desenvolvimento de uma tipologia que possibilite uma maior adequação da organização ao seu ambiente. 
Por fim, é importante destacar que o alinhamento identificado entre as três análises realizadas no presente estudo de forma a contribuir com a identificação das relações entre os estilos culturais de Paz e Mendes (2009) e estratégias competitivas de Miles e Snow (2003).

\section{CONCLUSÕES}

Após a análise e discussão dos resultados, pode-se, realizar algumas considerações conclusivas de forma a possibilitar atingir o objetivo geral proposto para realização do presente estudo que buscou relacionar os estilos culturais de Paz e Mendes (2009) com as tipologias de estratégias genéricas de Miles e Snow (2003).

Desta forma, ao se estudar os estilos culturais presentes nas IES identifica-se o predomínio de três estilos culturais dos quatro propostos por Paz e Mendes (2009), destes estilos culturais destacaram-se os estilos empreendedores e afiliativos. Esses resultados demonstram uma abertura cultural dessas IES em relação ao nível de maturidade de seus funcionários, que é percebido por meio de políticas que favorecem o crescimento e a inovação nestas instituições.

Quanto às tipologias estratégicas que foram adotadas pelas IES estudadas, observou-se a ocorrência das quatro estratégias propostas por Miles e Snow (2003), dentre estas estratégias destaca-se a predominância das estratégias prospectivas e defensivas. Este resultado demonstra a predominância de dois grupos de IES com características distintas, de um lado se tem organizações que se posiciona de forma inovadora com foco no processo de aprendizagem e um segundo grande grupo de IES mais conservador, apresentando centralidade decisória e eficiência dos processos internos.

Em relação a influência da cultura organizacional nas estratégias das IES do RN, os resultados da análise preditiva de correlação canônica indicaram uma forte relação entre os estilos culturais com as tipologias estratégicas, resultados estes confirmados com a correlação que indicam, além da influência da cultura em relação às estratégias, a influência da estratégia em relação a cultura, demonstrando a casualidade entre as duas dimensões que o modelo estatístico permite identificar. Outras análises estatísticas realizadas no estudo para atestar as relações entre cultura e estratégia foram à correlação de Pearson e a comparação entre a proposta teórica realizada no presente estudo com os resultados encontrados na pesquisa que indicaram $84 \%$ de conformidade.

Assim, reafirma-se de forma conclusiva a relação existente entre os estilos culturais de Paz e Mendes (2009) com as tipologias estratégicas de Miles e Snow (2003) nas IES do RN. Os resultados encontrados no presente estudos estão em conformidade aos estudos desenvolvidos por Degenhardt (2006), Conant, Monkwa e Varadajan (1990), Green (1988), Shrivastava (1985), Quinn e Grath (1985), Schein (2009), Porter (1986), Mintzberg; Ahistrand e Lampel (2000), Miles e Snow (2003) e Mintzberg (2003), apresentado assim, a consistência teórica do presente estudo.

O presente estudo pode proporcionar ao processo decisório das organizações estudadas, de maneira a despertar nos gestores uma atenção as suas características culturais, mesmo se apresentado dificuldades de modificá-las, pelo menos estes podem desenvolver estratégias alinhadas às estas características culturais identificadas.

Esta pesquisa deixa como recomendações para estudos futuros, a realização de pesquisas longitudinais para identificar a evolução natural da cultura e estratégias adotadas pelas IES do 
RN, além da realização de estudos para comparar os resultados desta pesquisa com a realidade de outras regiões do país.

Por fim, fica ainda como recomendações de estudos futuros, identificar outros fatores organizacionais que possam influenciar as relações entre cultura e estratégia organizacional, de forma a identificá-las no plano macro em relação a outros fatores pertencentes ao ambiente organizacional e sua interação com seus agentes. Para elaboração destes estudos se propõe o uso de modelos causais que permitam identificar múltiplas relações de interdependências.

\section{REFERÊNCIAS}

1. ALMEIDA, Martinho I. R. Manual de planejamento estratégico. São Paulo: Atlas, 2001.

2. CARVALHO, Carlos Eduardo; RONCHI, Carlos César. Cultura organizacional: teoria e pesquisa. Rio de Janeiro: Fundo de Cultura, 2005.

3. COLLIS, Jill; HUSSEY, Roger. Pesquisa em administração: um guia prático para alunos de graduação e pós-gradução. 2. ed. Porto Alegre: Bookman, 2005.

4. CONANT, J. S.; MOKWA, M. P.; VARADARAJAN, P. R. Strategic types, distinctive marketing competencies and organizational performance: a multiple measures-based study. Strategic Management Journal, v. 11, n. 5, p. 365-383, sep. 1990.

5. DAFT, Richard L. Organizações: teorias e projetos. São Paulo: Thomson, 2002.

6. D'ANJOUR, Miler Franco. As relações da cultura organizacional com as estratégias nas instituições de ensino superior do rio grande do norte. Dissertação (PPGA) - Universidade Potiguar, Natal, 2010.

7. DEGENHARDT, Victor Werner. Estratégia competitiva e cultura organizacional: um estudo exploratório do estágio atual da indústria brasileira de equipamentos mecânicos pesados. Dissertação (PPGA) - Pontifícia Universidade Católica de São Paulo, São Paulo, 2006.

8. DUTRA, Joel Souza; FLEURY, Maria Tereza Leme; RUAS, Roberto. Competências: Conceito, Métodos e Experiências. São Paulo: Ed. Atlas, 2006.

9. HAIR JR, Joseph F. et al. Análise multivariada de dados. 6. ed. Porto Alegre: Bookman, 2009.

10. KELM, Martinho Luís. Indicadores de performance em instituições universitárias autogeridas : uma contribuição à gestão por resultados. Tese (PEP) - Universidade Federal de Santa Catarina, Florianópolis, 2003.

11. MENDES, Ana M. Bezerra; PAZ, Maria das G. Torres da; Gabriel, lannis. Configurações de poder organizacional e estilos de caráter. Psicologia: organizações e trabalhos, Florianópolis, v. 1, n. 1, p. $141-186$, jan./jun. 2001.

12. MILES, R. E; SNOW, C. C. Organizational strategy, structure, and process. Stanford. Stanford Business Books, 2003.

13. MINTZBERG, Henry; AHLSTRAND, Bruce; LAMPEL, Joseph. Safári da estratégia: um roteiro pela selva do planejamento estratégico. Porto Alegre: Bookman, 2000.

14. MINTZBERG, Henry. Criando organizações eficazes: estruturas em cinco configurações. 2. ed. São Paulo: Atlas, 2003.

15. MINTZBERG, H; ROSE, J. Strategic management upside down: tracking strategies at MacGill 
University from 1829 to 1980. Canadian Journal of Administrative Science. v. 20, n. 4. p. 270-290, Dec., 2003.

16. MINTZBERG, Henry et al. O processo da estratégia. 4. ed. Porto Alegre: Bookman, 2006.

17. MOREIRA, Cristiane Rosa. A relação entre estratégia e performance nas instituições de ensino superior privadas na Região Sul. Dissertação (PPGA) - Universidade Federal de Santa Maria, Santa Maria, 2007.

18. MORGAN, Gareth. Imagens da organização. São Paulo: Atlas, 1996.

19. PAZ, Maria das G. Torres da; MENDES, Ana M. Bezerra. Estilos de funcionamento organizacional. In: SIQUEIRA, Mirlene Maria Matias (Org.). Medidas do comportamento organizacional: ferramenta de diagnóstico e de gestão. Porto Alegre: Artmed, 2008.

20. PORTER, Michael E. Competição: estratégias competitivas essenciais. 9. ed. Rio de Janeiro: Campus, 1999.

21. PUENTE-PALACIOS, K. E. Influências das características pessoais e ambientais na avaliação do clima social do trabalho. Revista de Administração, v. 30, n. 3, p.73-79, jul./set. 1995.

22. QUINN, R. E.; The transformation of organizational cultures. In. FROST, P. J. (Org.). Organizational culture. Beverly Hills: Sage, 1985.

23. SCHEIN, Edgar H. Cultura organizacional e liderança. São Paulo: Atlas, 2009.

24. SCHNEIDER, William E. Uma alternativa à reengenharia. Rio de Janeiro: Record, 1996.

25. SHRIVASTAVA, Paul. Integrating strategy formulation with organizational culture. Journal of Business Strategy, v. 5, n. 3, p. 103-111, Winter, 1985.

26. TONI, Jackson De. Em busca do planejamento governamental do século XXI: novos desenhos. In: REPETTO, Fabio et al. Reflexões para Ibero-America: planejamento estratégico. Brasília: ENAP, 2009. p. $21-36$.

27. VIZEU, Fabio; GONÇALVES, Sandro Aparecido. Pensamento Estratégico: origem, princípios e perspectivas. São Paulo: Atlas, 2010. 